\title{
La nanotecnología en México: un desarrollo incierto
}

\section{Nanotechnology in Mexico: an uncertain development}

\author{
Edgar Záyago-Lau \\ Guillermo Foladori*
}

\begin{abstract}
In this article we explore the incorporation of nanotechnology in the scientific and technological development in Mexico. We show that, although Mexico could be ranked in second place in this area in Latin America, the lack of a clear stimulation policy and the need of research centres for bilateral external support have created an uncertain future for a sustainable and articulated development of nanotechnology that would become the base of the next industrial revolution.
\end{abstract}

Keywords: nanotechnology, nano-science, Mexico, science and technology.

\section{Resumen}

En este artículo exploramos la incorporación de la nanotecnología en el desarrollo científico y técnico de México. Mostramos que, no obstante su probable segundo lugar en el ámbito latinoamericano, la falta de una clara política de promoción, y la necesidad por parte de los centros de investigación de apoyos externos bilaterales, ha creado un futuro incierto para un desarrollo sostenido y articulado de una tecnociencia que promete constituir las bases de la próxima revolución industrial.

Palabras clave: nanotecnología, nanociencias, México, ciencia y tecnología.

*Universidad Autónoma de Zacatecas, México. Correos-e: edzlau@yahoo.com; fola@estudiosdeldesarrollo.net. 


\section{Introducción}

El objetivo de este trabajo es ilustrar la manera en que la nanotecnología se inserta en el proceso de desarrollo capitalista México. Hasta el momento existe una laguna importante en la literatura sobre el inventario de recursos y objetivos del desarrollo nanotecnológico mexicano. Este trabajo pretende contribuir a reducir esa laguna, y lo dividimos en cinco partes. La primera muestra algunas cuestiones básicas sobre la nanotecnología y su potencial para convertirse en la plataforma de la siguiente revolución industrial. La segunda muestra la paradoja de un discurso que enfatiza la necesidad del desarrollo de la ciencia y la tecnología (C\&T) como instrumento del desarrollo, y una política económica que ha mantenido el presupuesto público muy por debajo de las expectativas respecto de las orientaciones internacionales. La tercera parte analiza el potencial de las nanotecnologías en México en función de los principales indicadores que se utilizan comúnmente en materia de C\&T. La cuarta realiza un diagnóstico de los esfuerzos de los centros de investigación por incorporarse a la revolución de las nanotecnologías. En la última parte se muestran algunas expectativas de integración de investigación y producción mediante la creación de parques industriales de alta tecnología. Las conclusiones resaltan la falta de una orientación pública planificada para encarar las nanotecnologías y los retos que esto supone; aunque algunos indicios aparecieron en 2009.

\section{Nanotecnología: algunas cuestiones básicas}

Dar una definición consensuada de la nanotecnología es una tarea difícil, ya que existen un sinnúmero de definiciones que utilizan diferentes criterios. Parece, no obstante, que existe un acuerdo en cuanto a que la nanotecnología implica una novedad revolucionaria, pues permite manipular y modificar la materia en la escala nanométrica (atómica, molecular y macromolecular).

De ahí que, inicialmente, debamos partir del entendimiento del tamaño para poder definir a la nanotecnología. A saber, un nanómetro representa una unidad de longitud del orden de una mil millonésima parte de un metro. Existen varios ejemplos, usados con frecuencia por los nanotecnológos para ilustrar el tamaño del que se habla, como la referencia de un cabello humano que contiene alrededor de 80,000 nanómetros (nm) de longitud; el virus del viH, causante del síndrome de inmunodeficiencia adquirida (SIDA), puede medir $60 \mathrm{~nm}$; una bacteria puede llegar a medir $20 \mathrm{~nm}$ y el tamańo de un nanotubo de carbono puede tener un diámetro de sólo $1 \mathrm{~nm}$. 
La Oficina Nacional de Coordinación de la Nanotecnología (ONCN) de Estados Unidos, define la nanociencia como aquélla que "involucra la investigación y el descubrimiento de nuevas características y propiedades de materiales en la nanoescala, cuyo rango va de 1 a 100 nanómetros (nm)"; y la nanotecnología como "la manera en que los descubrimientos en la nanoescala son puestos a trabajar" (2009: 2).

En contraste, existen otras definiciones que son un poco más ilustrativas en cuanto a las implicaciones de la nanotecnología. Por ejemplo Miller y Senjen explican lo siguiente:

\begin{abstract}
El término "nanotecnología" no describe a una sola técnica sino que engloba a una serie de tecnologías que operan a escalas de los componentes básicos de los materiales biológicos y manufacturados -es decir, a "nanoescala". Se ha definido provisionalmente a la nanotecnología como toda tecnología relacionada con materiales, sistemas y procesos que operan a una escala de 100 nanómetros (nm) o menos. Los nanomateriales han sido definidos como aquéllos que tienen una o más dimensiones que miden $100 \mathrm{~nm}$ o menos, o que tienen al menos una dimensión a esta escala que afecta el comportamiento y las propiedades de los materiales (2008: 27).
\end{abstract}

Otras definiciones contienen elementos similares sobre el comportamiento novedoso de la materia a partir de manipular la nanoescala:

[la nanotecnología es] El diseño, caracterización, producción y aplicación de estructuras, componentes y sistemas creados por la manipulación controlada de tamańo y forma en la escala nanométrica (atómica, molecular y macromolecular) que produce estructuras, componentes y sistemas con al menos una característica o propiedad novedosa o superior (Bawa et al., 2005: 151).

La Real Society y The Royal Society and the Royal Academy of Engineering (RS\&RAE, 2004: 8) definen la nanotecnología de manera similar a las anteriores, aunque elabora un poco más sobre el origen de las propiedades novedosas en la nanoescala, al decir que los materiales a dicha escala (1-100 nm) tienen una superficie (por unidad de masa) más amplia, lo que viabiliza cambios en su reactividad química: $y$, también, al enfatizar el tamaño en sí, y explicar que a tal escala la materia presenta efectos cuánticos magnéticos, ópticos y eléctricos diferentes. Por su parte, la Administración Nacional de Aeronáutica y del Espacio (NASA) de Estados Unidos define la nanotecnología como "la creación de materiales funcionales, componentes y sistemas mediante el control de la materia en la nanoescala (1-100 nanometros), y la explotación de fenómenos novedosos y nuevas propiedades (físicas, químicas, biológicas, mecánicas, eléctricas...) en esa escala" (NASA, s/f). Por esta razón, la nanotecnología se expone como una tecnología revolucionaria, puesto que marca el desarrollo 
de nuevos materiales o el descubrimiento de propiedades novedosas en materiales ya conocidos. Los materiales en la nanoescala presentan propiedades de resistencia, reactividad, conductibilidad, flexibilidad, durabilidad y toxicidad diferentes a los mismos materiales en tamaño mayor.

En consecuencia, podemos dar cuenta de que la nanotecnología no es una tecnología disciplinaria, particularmente si hablamos de su relación con la ciencia de materiales; más bien, se trata de una tecnología multidisciplinaria, que implica el entendimiento de la física, la química, la electrónica, la dinámica de fluidos y muchas otras ingenierías. Lo anterior viabiliza la función de la nanotecnología como una tecnología habilitadora que se pueda usar en cualquier sector industrial o rama productiva. De ahí que se le considere la plataforma de la revolución industrial del siglo xxI.

En la actualidad ya se observa el rápido crecimiento del mercado de esta tecnología revolucionaria. De acuerdo con algunas proyecciones, se estima que el mercado de las nanotecnologías llegará a los 1.5 mil millones de dólares (sin considerar los semiconductores) (Científica, 2007). En consecuencia, varios países han decidido, seducidos por las prospectivas de ganancia, invertir ampliamente en el desarrollo de estas tecnologías. Sólo en Estados Unidos se ha invertido, desde 2001, un estimado de 12 mil millones de dólares en investigación y desarrollo (I\&D) de esta tecnología (NNI, 2009: 5). México, al igual que otros países latinoamericanos, han seguido el camino con esfuerzos más modestos. El camino de las nanotecnologías en México es lo que se intenta ilustrar en el contenido de las secciones subsiguientes.

\section{La política de ciencia y tecnología en México}

La política de ciencia y tecnología $(C \& T)$ en México se inserta en el proceso de transición hacia la economía del conocimiento, como lo muestran los procedimientos y planeación de la política pública en la materia. Este objetivo lo promueven organismos internacionales como una receta técnica para impulsar el desarrollo. De acuerdo con organizaciones como el Banco Mundial (вм) y la Organización para la Cooperación y el Desarrollo Económico (OCDE), la C\&T se ve como el detonador más importante en el proceso de transformación productiva de las economías emergentes y en vías de desarrollo. El Banco Mundial, por ejemplo, dice:

La economía del conocimiento es aquélla en la que los factores de conocimiento adquieren, de manera deliberada, más importancia que el capital y los factores de trabajo; y donde además, la cantidad y sofisticación del conocimiento que permean a las actividades sociales y económicas alcanzan niveles muy altos (2007: 14). 
De acuerdo con metodologías del Banco Mundial para medir la capacidad de los países para transitar a una economía del conocimiento, y según la clasificación de los países en relación con sus capacidades en la economía del conocimiento, publicado en 2008, México se encuentra ubicado en la posición 59, debajo de países como Dinamarca (1), Estados Unidos (9), Corea del Sur (31) pero también de Brasil (54) (KEI, s/f). Se argumenta que el deficiente desempeño económico de México es resultado de la falta de acumulación de conocimiento y de la poca difusión del mismo (OCDE, 2009). Por estas razones, la política de C\&T de México se vincula con la creación de una economía del conocimiento competitiva.

Las referencias de la política pública mexicana del desarrollo a los pilares de la economía del conocimiento son claras. En el Plan Nacional de Desarrollo (PND) 2007-2012 encontramos cinco ejes rectores que en teoría promoverán el crecimiento económico del país, entre éstos hay uno dirigido a lograr el desarrollo de una economía competitiva y generadora de empleos. De manera complementaria en el Programa Especial de Ciencia y Tecnología (РЕСYT) 2008-2012 se establece que tanto la ciencia como la tecnología "juegan un papel preponderante como variables estratégicas del cambio estructural para el desarrollo del país [con el objeto de] potenciar la productividad y competitividad de la economía mexicana para lograr un crecimiento económico sostenido y acelerar la creación de empleos" (РЕСтT, 2008: 3).

En junio de 2009, ciertos cambios en La Ley de Ciencia y Tecnología hicieron más clara la ya explícita orientación de la C\&T y la innovación en México para el incremento de la competitividad. En dicha ley se asienta el fomento a la C\&T como una expresión del crecimiento económico liderado por la empresa, pero apoyado por el sector científico y el gobierno. En el cuerpo de la Ley de Ciencia y Tecnología publicada el 12 de junio de 2009, en el Diario Oficial de la Federación (DOF), se puede leer, entre otros, el siguiente propósito:

Fomentar el desarrollo tecnológico y la innovación de las empresas nacionales que desarrollen sus actividades en territorio nacional, en particular en aquellos sectores en los que existen condiciones para generar nuevas tecnologías y lograr mayor competitividad. Incorporar el desarrollo tecnológico y la innovación a los procesos productivos y de servicios para incrementar la productividad y la competitividad que requiere el aparato productivo nacional (2009: 1).

Lo expuesto anteriormente confirma la motivación detrás de la política de C\&T en México, es decir, la búsqueda constante de competitividad mediante el conocimiento. No obstante, la realidad refleja resultados diferentes a las expectativas contenidas en los planes, resaltando la falta de recursos para llevar a cabo las propuestas. Nos referimos concretamente 
a lo relacionado con el financiamiento que el gobierno federal destina al desarrollo de C\&T. El gasto en este rubro representa las erogaciones en cuenta corriente, infraestructura, pago de pasivos y deuda pública destinada a la C\&T. De acuerdo con el вм, los países deben destinar, al menos, entre $1 \%$ y $1.5 \%$ del producto interno bruto (PIB) al desarrollo de C\&T. Sin embargo, México se ha quedado muy por debajo de lo recomendado. Es más, desde 1998 se ha verificado una tendencia hacia la disminución del presupuesto en este rubro. La tendencia a la baja en el gasto en $C \& T$ de México se ha mantenido en años recientes. En el año 2008, el presupuesto federal destinado a este rubro solamente alcanzó, como porcentaje del PIB, $0.32 \%$ y un estimado para 2009 de un magro 0.33\% (Ruíz, 2009).

La falta de recursos para financiar la C\&T en México ha tenido repercusiones en otros indicadores que muestran la falta de encadenamiento entre el diseño de la política pública de C\&T y su operacionalización. Uno de estos indicadores es el gasto destinado a la investigación y desarrollo experimental (IDE) como porcentaje del PIB. Este índice lo creó la OCDE, en 1963, y se incluye en el Manual de Frascati, cuya última edición se publicó en 2002. El índice se encarga de medir las actividades directamente relacionadas con la I\&D (OCDE, 2002: 30). El gasto en IDE representa el último eslabón, pero es indispensable en la cadena del conocimiento, previo al patentado y la comercialización. En el gasto en IDE no se incluyen rubros relacionados con la formación y enseñanza, actividades afines industriales y tampoco gastos administrativos. Por consiguiente es un indicador de lo que realmente se destina a la innovación en un país. México sólo dedica $0.50 \%$ del PIB en IDE, esto es menor de lo que destinan otras economías de similar tamańo como Brasil, con 1.02\%; o desarrolladas como Alemania con 2.53\%; Estados Unidos con 2.62; Corea del Sur con 3.23\% y Japón con 3.39\% (OCDE, 2008).

El coeficiente de inventiva es otro indicador importante relacionado con las actividades de C\&T y en el cual, para el caso de México, también se ha reflejado la reducción de presupuesto. Este coeficiente se refiere al número de patentes obtenidas por cada 10,000 habitantes. El indicador es útil para comparar el nivel de innovación que existe entre diferentes países. Por ejemplo, el coeficiente para México es de 0.05, para Brasil 0.21, para Estados Unidos 6.45, para Corea del Sur 21.89 y para Japón 28.84 (Conacyt, 2007). De nueva cuenta se refleja la pérdida de competencia en este rubro dedicado a medir el nivel de desarrollo del conocimiento.

Otra guía que da cuenta del estado real de la C\&T en México es el índice global de innovación (IGI) que toma en cuenta, además de factores tradicionales como el gasto en $I \& D$, la preparación de los recursos humanos, el modelo de innovación en los negocios, las capacidades organizativas 
y operacionales, la fortaleza institucional y la capacidad de innovación de la sociedad. Instituciones especializadas en el estudio de la competitividad argumentan que todos estos elementos representan aspectos conducentes e indispensables para lograr un incremento de la misma (Insead, 2009: 7). En 2009 el IGI coloca a México en el lugar 61, por debajo de Brasil en el lugar 50; Japón en el 9; Corea del Sur en el 6; Alemania en el 2 y Estados Unidos que se encuentra en el primer lugar (Insead, 2009).

Es de resaltar, además, que la continua búsqueda de la competitividad no se traduce necesariamente en la reducción de la inequidad y de la consecuente pobreza. El coeficiente de Gini muestra que los países con una mejor posición en la clasificación de la competitividad global no necesariamente mantienen una menor desigualdad social. El coeficiente de Gini mide la desigualdad del ingreso en los habitantes de los países y, para hacerlo, se basa en una curva de distribución que va del valor 0 al 1 . El valor 0 corresponde a la perfecta igualdad, donde todas las personas mantienen los mismos ingresos. En contraste, el valor 1 corresponde a la perfecta desigualdad, es decir, donde una persona tiene todos los ingresos y los demás ninguno. En este sentido vale comentar que resalta el caso de Estados Unidos, que es el país más competitivo del planeta de acuerdo con el ICM (índice de competitividad mundial), pero que mantiene una tasa de desigualdad en el ingreso sobresaliente, aproximadamente de 0.41 puntos, por encima de otros países que se encuentran más abajo en la clasificación del ICM pero que mantienen valores de desigualdad más reducidos como es el caso de España, Francia, Japón y Alemania (UNDP, 2008). En el caso de México encontramos que existe un coeficiente de desigualdad de 0.46 , que de acuerdo con la última evaluación de pobreza, se traduce en $42.6 \%$ de personas en México, alrededor de 44.7 millones, que viven en la miseria (Coneval, 2008). Brasil es otro caso que llama la atención ya que sus indicadores de $C \& T$ son mejores que otras naciones de similar tamaño, como Corea del Sur y México; no obstante, Brasil mantiene una desigualdad en el ingreso mayor que estos países.

Otra forma de medir las diferencias de la política de C\&T en México con respecto a la de otros países es el número de patentes que se registran. El registro de patentes es el eslabón previo a la comercialización de un producto derivado de la I\&D, y también representa un objetivo crucial en la transición a la economía del conocimiento. Este proceso se realiza en el marco legal de la Organización Mundial de la Propiedad Intelectual (OMPI), en las oficinas de registro que existen en cada país. De esta forma el inventor, ya sea individuos o empresas, decide en qué país es más conveniente obtener el registro de patente para su producto o proceso. Una vez que se tiene el derecho de patente, éste otorga exclusividad de comercialización hasta por 20 años, dependiendo del sector (wIPO, 2008: 


\section{Cuadro 1}

Número de patentes registradas por país en la United States Patent and Trademark Office (2004-2007)

\begin{tabular}{lrrrr}
\hline \multicolumn{1}{c}{ Pais } & 2004 & 2005 & 2006 & 2007 \\
\hline México & 86 & 80 & 66 & 55 \\
Brasil & 106 & 77 & 121 & 90 \\
Chile & 15 & 9 & 14 & 25 \\
Corea del Sur & 4,428 & 4,352 & 5,908 & 6,295 \\
Alemania & 10,779 & 9,011 & 10,005 & 9,501 \\
Japón & 35,348 & 30,341 & 36,807 & 33,354 \\
Estados Unidos & 84,271 & 74,637 & 89,823 & 79,527 \\
China & 404 & 402 & 661 & 772 \\
India & 363 & 384 & 481 & 546 \\
Francia & 3,380 & 2,866 & 3,431 & 3,130 \\
\hline
\end{tabular}

Fuente: Elaboración propia con datos de la OMPI (2008).

62). La oficia de la Ompi en Estados Unidos, la United States Patent and Trademark Office (UPSTO), es quien recibe mayor número de solicitudes de registro, porque se encuentra en el país con el mercado más grande del mundo. Es interesante observar el número de patentes registradas por inventores residentes en México y comparar la cantidad de patentes registradas en Estados Unidos por inventores de otros países (cuadro 1).

Se observa una caída paulatina en el número de patentes registradas por México, circunstancia que se puede relacionar con la disminución en los fondos dedicados al desarrollo de C\&T.

La política que promueve la transición a la economía del conocimiento y que busca incrementar la competitividad, también genera inequidad en el desarrollo de la C\&T en los estados de México. La desigualdad entre los estados en cuanto a infraestructura de C\&T, innovación y otros factores es tan grande y centralizada en los centros urbanos, que la OCDE (2009) ha reconocido que existen diferentes Méxicos en este rubro. En este sentido se puede argumentar que México es un caso representativo de la desarticulación entre competitividad e inequidad, ya que desde la mitad de los ańos ochenta hasta mediados de los noventa la competitividad se incrementó significativamente, pero también la inequidad con el coeficiente de Gini, que pasó de 0.49 a 0.55 , respectivamente (Delgado e Invernizzi, 2002). Como consecuencia las dinámicas de los efectos desiguales en el desarrollo de C\&T de México también han permeado el desarrollo de la nanotecnología y la nanociencia, situación que se analiza en la siguiente sección. 


\section{La política mexicana de C\&T en relación con la nanotecnología}

La política mexicana en lo que se refiere a nanotecnología refleja los objetivos y la dinámica contenida en la política en $C \& T$, es decir, la búsqueda de la competitividad y el encadenamiento productivo para mejorar las ventajas comerciales. En esta configuración, México mantiene una segunda posición en el desarrollo de la nanotecnología en relación con otros países de Latinoamérica. México, después de Brasil, es uno de los líderes en la región de acuerdo con el número de instituciones que realizan investigación, la infraestructura creada, el número de publicaciones académicocientíficas, los convenios internacionales y la cantidad de recursos humanos trabajando con nanotecnología (Foladori, 2006; OEI, 2007).

El interés por desarrollar la nanotecnología en México se expresó a partir de 2001 en el Programa Especial de Ciencia y Tecnología (PECYT) 2001-2006. En este plan se expone la nanotecnología como una tecnología estratégica y con un potencial de desarrollo importante, especialmente en el sector energético mediante las capacidades de investigación y de infraestructura del Instituto Mexicano del Petroleo (IMP) (PECYT, 2001: 95). El posicionamiento de la nanotecnología como un área estratégica de desarrollo para México se reafirmó en el PECYT 2008-2012, donde se lee que esta tecnología, junto con otras tecnologías emergentes, son fundamentales para "contribuir a mejorar el nivel de vida de la sociedad y lograr una mayor competitividad" (PECYT, 2008: 25). No obstante, hasta la fecha no existe una iniciativa nacional o un plan nacional de nanotecnología. Tampoco hay una oficina o consejo administrativo que marque los objetivos y dirección que la nanotecnología debe seguir de acuerdo con el contexto de México. Esto implica grandes retos ya que al no tener objetivos claros y una dirección determinada, el desarrollo de la nanotecnología en México es ambiguo. No hay directivas claras de las áreas que se deben apoyar, se carece de bases de datos que faciliten el trabajo en redes, no existen mecanismos que eviten que se dupliquen los esfuerzos y tampoco contamos con políticas que favorezcan la utilización compartida y coordinada de infraestructura de laboratorio.

Se sabe, a partir de un informe elaborado por la Secretaría de Economía, que el Consejo Nacional de Ciencia y Tecnología (Conacyt) financió, entre 1998 y 2004, aproximadamente 152 proyectos de investigación relacionados con nanotecnologías; el monto total se estima en 14.4 millones de dólares (sE, 2008). En 2006, el Conacyt lanzó una convocatoria para recibir propuestas para crear laboratorios nacionales de C\&T. En esta convocatoria se aprobó la creación de dos laboratorios de investigación en nanotecnología. El primero, el Laboratorio Nacional de Nanotecnología (Nanotech), se localiza en Chihuahua, en el Centro de Investigación en 
Materiales Avanzados (Cimav), y, el segundo, el Laboratorio Nacional de Investigaciones en Nanociencias y Nanotecnología (Linan), tiene sede en San Luis Potosí, en el Instituto Potosino de Investigación Científica y Tecnológica (IPICYT). Cada uno de estos laboratorios recibió alrededor de 20 millones de pesos para su desarrollo en el año 2006 (Conacyt, 2006a).

$\mathrm{La}$ ausencia de una iniciativa nacional no permite recopilar información confiable en cuanto al monto total que se gasta en nanotecnología en México. No obstante, si tomamos lo que se estima que se gastó en México para el periodo 1998-2004 en I\&D de nanotecnología, aproximadamente 14.4 millones de dólares, podremos darnos cuenta que en comparación con lo que invierten los países que están a la vanguardia es una cantidad muy pequeña. En el cuadro 2 se muestra el gasto estimado en nanotecnología sólo en 2008 por parte de algunos países.

\section{Cuadro 2}

Inversión en I\&D en nanotecnología en países líderes (2008)

\begin{tabular}{lc}
\hline $\begin{array}{c}\text { País } \\
\text { (estimado) }\end{array}$ & $\begin{array}{c}\text { Inversión en millones de dólares } \\
\text { Mundo }\end{array}$ \\
Unión Europea & 7,849 \\
Estados Unidos & 2,440 \\
Japón & 1,821 \\
Rusia & 1,128 \\
Alemania & 1,076 \\
China & 541 \\
Corea del Sur & 510 \\
Reino Unido & 350 \\
Taiwán & 184 \\
India & 97 \\
\hline
\end{tabular}

Fuente: Elaboración propia con información de Científica (2009).

Esto nos da una idea de los países que son los grandes jugadores en nanotecnología y los que terminan moldeando las tendencias competitivas en el mercado mundial. Es de resaltar que a diferencia de México, varios de estos países líderes en nanotecnología cuentan con iniciativas nacionales en la materia, lo que les permite identificar sectores de oportunidad y espacios de mercado a explotar.

La diferencia en recursos y organización del caso mexicano para desarrollo de nanotecnología no es solamente visible en relación con las economías más industrializadas. También se puede observar una diferencia de México con respecto a otras economías emergentes que han ordenado la I\&D de la nanotecnología en una iniciativa nacional y que, de manera paralela, han incrementado el financiamiento en este rubro. Un indica- 


\section{Cuadro 3}

Artículos publicados acerca de nanociencias y nanotecnologías en países con economías emergentes

\begin{tabular}{lccc}
\hline País & Periodo 2000-2003 & Periodo 2004-2007 & Crecimiento (\%) \\
\hline México & 877 & 1,505 & 71.6 \\
China & 12,870 & 36,910 & 186.8 \\
Corea del Sur & 4,532 & 11,003 & 142.8 \\
India & 2,652 & 6,778 & 155.6 \\
Brasil & 1,930 & 3,203 & 66 \\
Argentina & 607 & 898 & 47.9 \\
\hline
\end{tabular}

Fuente: datos del Science Citation Index (scI) (2008).

dor que se puede utilizar para ilustrar la mencionada diferenciación es la cantidad de artículos publicados relacionados con la nanotecnología, es decir, la producción académico-científica al respecto (cuadro 3).

Podemos observar que México se encuentra muy por debajo de otras economías emergentes en la publicación de artículos académico-científicos en temas relacionados con nanotecnología. Esto a pesar de que las publicaciones en México han crecido, en promedio, más que las publicadas por otros países con economías de similar tamaño -como Brasil-, aun así, éste último supera al primero con el doble de publicaciones.

La política mexicana en nanotecnología reproduce los efectos centralistas de la política en C\&T. Lo anterior conlleva a que la mayor parte de los recursos humanos y de infraestructura en nanotecnología se concentren en los grandes centros urbanos del país, sobre todo el Distrito Federal, es decir, la entidad más competitiva de México (Imco, 2008). Y, precisamente, las universidades y laboratorios localizados en esta ciudad son los que aportan el mayor número de publicaciones relacionadas con la nanotecnología. Si se consideran las publicaciones acumuladas en el tema para el periodo 1995-2007, encontramos que la Universidad Nacional Autónoma de México (UnAm) produjo 40\% de los artículos indexados, y el Centro de Investigaciones y de Estudios Avanzados del Instituto Politécnico Nacional (Cinvestav) publicó $16.44 \%$ en el mismo periodo (Robles-Belmont et al., 2008). Se espera que tal circunstancia cambie, al menos en lo relacionado con la producción científico-académica de las nanotecnologías, particularmente después de la creación del Nanotech en Chihuahua y el Linan en San Luis Potosí. No obstante, para el periodo que se estudia, la centralización del número de publicaciones por institución es evidente. Una contabilidad de publicaciones en nanociencia y nanotecnología entre 1997 y 2005 nos muestra que la UNAM y el Cinvestav concentran más de la mitad de todas las publicaciones sobre nanotecnología en México (Robles-Beltmont et al., 2008). Los demás centros de investigación y universidades contribuyen 
con un porcentaje muy bajo en relación con el total. Además, de nueva cuenta se observa el patrón en el cual las entidades más competitivas, de acuerdo con el Instituto Mexicano para la Competitividad (Imco), son las que mantienen los mejores centros de investigación y universidades trabajando en nanotecnología. En contraste, no se encuentra infraestructura significativa ni recursos humanos calificados en el área en estados como Hidalgo, Chiapas, Guerrero, Oaxaca y Tlaxcala, entidades localizadas en los últimos lugares de competitividad estatal (Imco, 2008).

En el universo de publicaciones relacionadas con la nanotecnología y la nanociencia en México, se han identificado las áreas donde las instituciones mexicanas se han especializado (Robles-Beltmont et al., 2008). El área de ciencia de los materiales es la que concentra el mayor número de publicaciones relacionadas con estos temas. Aunque esto puede ser relativo, ya que la ciencia de los materiales incluye el manejo de elementos de física, química, electrónica, dinámica de fluidos y diversas ingenierías. En términos de patentes, y de acuerdo con la Organización de Estados Iberoamericanos (OEI) y su estudio La nanotecnología en Iberoamérica. Situación actual y tendencias, publicado en 2007, los títulos de patentado obtenidos por instituciones e investigadores radicados en México son pocos en comparación con otros países. En este estudio se utiliza la base de datos de la Organización Mundial de la Propiedad intelectual (wipo, por sus siglas en inglés), que contiene los títulos de patentes registrados de acuerdo con el Tratado de Cooperación de Patentes (РCT, en inglés). El PCT tiene validez legal en muchos países, lo que permite solicitar el registro de patente simultáneamente en varias oficinas.

En el mencionado estudio de la oEI se expone que España aventaja a las demás naciones de Iberoamérica con 369 títulos de patentes registrados, le sigue Brasil con 89; Portugal con 36, México con 28, Argentina con 12, Chile con 10, Panamá con nueve, Cuba con siete, Puerto Rico y Uruguay con dos, y Honduras y Venezuela con un registro cada uno. Si se considera sólo a los países latinoamericanos encontramos que México ocupa el segundo lugar después de Brasil en el patentado de nanotecnología, lugar que se ha refrendado en otras áreas científicas y económicas.

El Instituto Mexicano del Petroleo (IMP) es la institución que más registros mantiene ante el PCT, y éstos son sólo cuatro. Sin embargo, esto concuerda con la encomienda del РЕСтT (2001: 95) de vincular, en lo posible, el desarrollo de la nanotecnología en México con el sector energético. La UnAM es la segunda institución en cuanto al número de registros con dos. Pero en realidad la UNAM, al ser la universidad con la mayor cantidad de publicaciones en nanotecnología con 1,200 (RoblesBelmont et al., 2008), y llevar a cabo más de $50 \%$ de toda la investigación científica del país (Lopez y Roldán, 2009), pareciera quedarse corta en el 
registro de patentes en nanotecnología, particularmente en comparación con las instituciones con sede en otros países.

\section{Principales instituciones, centros y laboratorios de México que trabajan con nanotecnología}

En México existen varias instituciones, laboratorios, centros de investigación y educativos, universidades e institutos que trabajan con nanotecnología. Se calcula que existen 56 instituciones realizando actividades de investigación, más de 159 laboratorios y se estiman 340 líneas de investigación actualmente desarrolladas sobre nanotecnología y nanociencia; también se calcula que hay 449 investigadores trabajando en la temática de los cuales $29 \%$ se encuentran adscritos a centros de investigación Conacyt, $18 \%$ están en la UNAM, $15 \%$ en el IMP, $8 \%$ en el Instituto Politécnico Nacional (IPN) y $30 \%$ en otras 20 instituciones (SE, 2008).

Es importante mencionar que como resultado de la ausencia de una iniciativa nacional y de objetivos claros, los esfuerzos nanotecnológicos en México están dispersos y aislados. La infraestructura dedicada a la nanotecnología, representada por los laboratorios especializados, es una forma de identificar a los principales centros de investigación en el área en México (cuadro 4).

\section{Cuadro 4}

Instituciones con más laboratorios dedicados a la I\&D de nanotecnología en México

\begin{tabular}{lc}
\hline \multicolumn{1}{c}{ Institución } & Número de laboratorios \\
\hline CPI-Conacyt (no se incluye Cimav e Ipicyt) & 45 \\
UNAM (incluye facultades e institutos) & 23 \\
Cimav & 18 \\
IMP & 16 \\
IPN (incluye Cinvestav) & 7 \\
Ipicyt & 6 \\
Universidad de Sonora & 6 \\
Universidad de Guadalajara & 6 \\
Universidad Autónoma Metropolitana & 5 \\
Universidad Autónoma de Chihuahua & 3 \\
\hline
\end{tabular}

Fuente: Elaboración propia y complementada con SE (2008).

De acuerdo con particularidades técnicas, sólo los laboratorios de instituciones como la UNAM, el IMO, el Centro Nacional de Metrología (Cenam) y los de algunos centros públicos de investigación (CPI)-Conacyt, como el Cimav, el Ipicyt y el Centro de Investigaciones en Química 
Aplicada (CIQA), tienen capacidad para abordar los temas de frontera de la nanotecnología y la nanociencia (sE, 2008: 122).

El Centro de Investigación en Materiales Avanzados (Cimav), ubicado en Chihuahua, se ha convertido en uno de los actores más importantes en I\&D de la nanotecnología en México. En 2008 el Cimav inauguró el Nanotech (laboratorio nacional), con el propósito de poner al servicio de las instituciones y empresas mexicanas su competitividad y complementariedad para desarrollar aplicaciones específicas que devengan en productos industriales (Nanotechch, s/f). El Nanotech planea ser el centro de contacto de todas las actividades en nanotecnología de México con el extranjero, por lo que en 2009 se convirtió, luego de haber ganado un concurso de la Secretaría de Relaciones Exteriores con un proyecto de dos años, en el Punto Nacional de Contacto en Nanotecnología y Nuevos Materiales (PNCNM). Además, este laboratorio, en asociación con la Universidad Estatal de Arizona, encabeza el Cluster de Innovación en Nanotecnología en América del Norte, con un fondo a partes iguales de 16 millones de dólares para ser utilizados en cinco años (sE, 2008). Otro plan importante es la creación del Centro Trinacional de Nanotecnología (CTN), formado por Brasil, Argentina y México, y del cual el Cimav y el Nanotech serán sedes coordinadoras del proyecto (Quezada, 2009). Se espera que el Nanotech trabaje todas las áreas relacionadas con la nanotecnología, aunque tienen planeado poner énfasis en síntesis de materiales, caracterización de materiales, desarrollo de nuevos materiales y asesoría, capacitación y entrenamiento para empresas e institutos.

El Ipicyt, es otra institución clave de la nanotecnología mexicana y es sede del Linan (el otro laboratorio nacional), que tiene entre otros objetivos orientar sus investigaciones para formar recursos humanos competitivos y contribuir al desarrollo de empresas en la región competitivas internacionalmente (Linan, s/f). El Linan, al igual que el Nanotech del Cimav, se inauguró en 2008. Este laboratorio presume de una colaboración continua con instituciones, empresas, laboratorios y centros de investigación del país y del extranjero, que incluye entre otros a la NASA, el MIT, el United States Department of Comerce (USDC), la ShinShu University de Japón, la unam, el ImP, el Instituto Tecnológico de Estudios Superiores de Monterrey (ITESM), el Instituto de Física del IPN y muchas otras organizaciones. El Linan también ofrece la maestría y el doctorado en nanociencia y nanotecnología, que hasta el momento son de los pocos reconocidos en el área en el ámbito nacional en el padrón de excelencia de Conacyt (PNP). Sus líneas de investigación incluyen la síntesis de nanoestructuras y biomimética, los nanomateriales magnéticos y sus aplicaciones, la nanobiotecnología, la caracterización de biomateriales nanoestructurados, 
los nanocompuestos y los cálculos electrónicos de nanosistemas, entre otros (Linan, s/f).

Tanto el Nanotech como el Linan se presentan como los proyectos bandera de la nanotecnología en México. No obstante, por su reciente creación apenas en el 2008, su contribución en el grueso de la I\&D de la nanotecnología en México no es muy significativa. Empero, se espera que estos laboratorios aglutinen la mayor parte de los recursos específicos para la investigación nanotécnica. Existen, sin embargo, otras universidades y centros de investigación que ya han desarrollado investigación y líneas de experimentación en el área de nanotecnología; veamos a continuación algunas de las instituciones más sobresalientes.

La UNAM es la institución que concentra el mayor número de investigadores en nanotecnología y posiblemente los proyectos mas ambiciosos. Uno de ellos es el Programa Impulsa, creado con el propósito de fomentar la investigación multidisciplinaria enfocada a resolver problemas nacionales. Forma parte de este programa el Proyecto Universitario de Nanotecnología (Punta), aprobado en diciembre de 2004, que trabaja en el desarrollo de nanomateriales. Dicho proyecto comenzó con un millón de dólares anuales de financiamiento e incorpora a ocho entidades de la UNAM, que a su vez contienen un total de 30 investigadores expertos en el área, la gran mayoría localizados en la ciudad de México (Punta, 2004). Otro cuerpo importante relacionado con la investigación de la nanotecnología es la Red de Grupos de Investigación en Nanociencias (Regina), con más de 50 investigadores de ocho diferentes centros y unidades de la UNAM (Regina, s/f). En esta red existen varias líneas de investigación en nanociencia y nanotecnología: síntesis de nanopartículas por métodos coloidales, propiedades ópticas de nanoestructuras de impurezas en cristales inorgánicos, películas delgadas nanoestructuradas, orden atómico local en nanocristales, sistemas nanoestructurados con aplicación en catálisis, física computacional de nanomateriales, nanomáquinas y heteroestructuras, nanobiotecnología, nanomagnetismo y muchas otras más (Regina, s/f). Además, en marzo de 2008, el Centro de Ciencias en Materia Condensada (CCMC) de la unAm, localizado en Ensenada, Baja California, se convirtió en el Centro de Nanociencia y Nanotecnología (CNYN), otro actor importante en la I\&D de esta área en México.

El IMP, ubicado en el Distrito Federal, es una institución sobresaliente y sectorizada en el campo de la energía y mantiene planes de I\&D de nanotecnología ambiciosos. Hay que recordar que es uno los pocos centros de investigación que han logrado patentar en el área de la nanotecnología y, de hecho, el que más registros de patentes mantiene (OEI, 2007). Además, tiene un programa de entrenamiento académico en el área de ingeniería molecular. El IMP representa un foco de atención para 
la I\&D de nanotecnologías para el sector energético por su directa relación con Petróleos Mexicanos (Pemex). El reto para la nanotecnología del IMP es descubrir formas para hacer más eficiente el uso de los derivados del petróleo y buscar nuevas fuentes de energía, aunque actualmente su línea de investigación más fuerte en el área nano es en nuevos materiales y nanoestructuras (IMP, s/f).

El Cinvestav es otro actor importante en el desarrollo de la C\&T en México en general y en la nanotecnología en particular. Cuenta con varios campus en diferentes ciudades de México dedicados a la investigación de la nanotecnología, pero particularmente el Departamento de Física, en el Distrito Federal, cuenta con un laboratorio avanzado para el estudio de nanoestructuras semiconductoras. Adicionalmente, el Cinvestav imparte la maestría y el doctorado en nanociencias y nanotecnología con especialidad en nanomaeriales, nanodispositivos y nanosistemas y bionanotecnología (Cinvestav, s/f). Recordemos, además, que el Cinvestav es la segunda institución con el mayor número de publicaciones científicas relacionadas con la nanotecnología (Robles-Belmont et al., 2008).

El Centro Nacional de Metrología (Cenam), localizado en la ciudad de Querétaro, es un centro encargado de establecer estándares de medición, incluidas las normas relacionadas con la nanotecnología. Para tal propósito cuenta con laboratorios de alto nivel en metrología química, electrónica, física, mecánica y en materiales. Entre los objetivos del Cenam se encuentra promover el conocimiento y uso de la metrología para fortalecer la competitividad de la industria, la equidad en las transacciones comerciales, la salud, la protección al ambiente y la investigación científica (Cenam, s/ fa). Adicionalmente, el Cenam participa en el Tri-national Workshop on Standards for Nanotechnologies, conjuntamente con el National Institute of Measurement Standards (NIMS) de Canadá y el National Institute of Standards and Technology (NIST) de Estados Unidos. El primer seminario se llevó a cabo en 2007, y el tercero, en febrero de 2009, en la ciudad de Querétaro. El Cenam, el NIMs y el NIST trazaron varios objetivos en este último seminario, incluyendo el de analizar los riesgos sobre el medio ambiente, en el lugar de trabajo, sobre la salud, sobre la sociedad y para los consumidores de los productos que usan o se fabrican a partir de la nanotecnología (Cenam, s/fb). Esto último convierte al Cenam en el único instituto de ciencias exactas y naturales que hasta el momento ha mostrado una agenda para analizar los temas sociales relacionados con la nanotecnología en México.

Otra institución importante es la Benemérita Universidad Autónoma de Puebla (BUAP), que tiene una participación destacada en el área de semiconductores y es sede de la Red Internacional de Nanociencia y Nanotecnología, donde participan investigadores mexicanos de la misma 
BUAP, del Instituto Nacional de Neurología y Neurocirugía (INNN), de la Sección de Estudios de Posgrado e Investigación de la Escuela Superior de Ingeniería Mecánica y Eléctrica (SEPI-ESIME) del IPN, de la Universidad Autónoma Metropolitana-Iztapalapa (UAM-I), del Cinvestav de las unidades Zacatenco y Mérida, de la UNAM y del Instituto Nacional de Investigaciones Nucleares (ININ).

Mención aparte merece el Instituto Nacional de Astrofísica, Óptica y Electrónica (INAOE), que es sede del Laboratorio Nacional de Nanoelectrónica (LNN), ubicado en Puebla y que cuenta con dos cuartos limpios para fabricación de dispositivos y circuitos integrados. El LNN comenzó con la donación de una línea de fabricación de dispositivos y circuitos integrados por la compañía transnacional Motorola, Inc. El objetivo del INAOE es ser "enlace entre la investigación de alto nivel tecnológico y el sector industrial, lo cual redundará en el desarrollo de una industria electrónica nacional y coadyuvará a mejorar el entorno universidad-industria-gobierno mediante un proyecto autosustentable y competitivo" (INAOE, s/f). Podemos observar, de nueva cuenta, que la fuerza invisible de la competitividad también se encuentra presente en el caso del INAOE y del LNN.

Las mencionadas universidades, centros de investigación y laboratorios representan lo más avanzado en México en cuanto a la I\&D de nanotecnología. Además, son las instituciones que publican más en la materia, que mantienen el mayor número de posgrados relacionados con nanotecnología y también los que aglutinan el grueso de investigadores en México.

Es importante mencionar que todos los laboratorios nacionales en nanotecnología, Nanotech, Linan y LNN, hacen referencia explícita sobre incorporar sus capacidades y desarrollos nanotecnológicos a la trayectoria de la competitividad industrial. También las otras instituciones de la nanotecnología mexicana, aunque no lo mencionan explícitamente, dejan ver que sus planes de I\&D se encuentran en tal trayectoria.

Existen muchas otras universidades y centros que trabajan con nanotecnología en México. No obstante, su contribución en el área en temas como patentes, número de publicaciones, infraestructura (laboratorios del más alto nivel) y formación de recursos humanos no es tan amplia como los centros, laboratorios e instituciones antes señalados. La lista de estas universidades, laboratorios y centros de investigación incluye: el Centro de Ingeniería y Desarrollo Industrial (Cidesi); Centro de Innovación Aplicada en Tecnologías Competitivas (Ciatec), Centro de Investigación Científica de Yucatán, A.C. (CICY), Centro de Investigación Científica y de Educación Superior de Ensenada (CICESE), Centro de Investigación en Alimentación y Desarrollo, A.C. (CIAD), Centro de Investigación y Asistencia en Tecnología y Diseño del Estado de Jalisco, A.C. (Ciatej), Centro de Investigaciones Biológicas del Noroeste, s.c. (Cibnor), Centro 
de Investigación y Desarrollo Tecnológico en Electroquímica (Cideteq), Centro de Investigaciones en Óptica, A.C. (Cio), Centro de Tecnología Avanzada Querétaro, A.C. (Ciateq), Corporación Mexicana de Investigación en Materiales, s.A. de c.v. (Comimsa), Universidad Autónoma de Ciudad Juárez (UACJ), Universidad Autónoma de Nuevo León (UANL), Universidad Autónoma de San Luis Potosí (UASLP), Universidad Autónoma de Yucatán (UAY), Universidad Autónoma del Estado de Hidalgo (UAEH), Universidad de Guanajuato (Ug), Universidad Michoacana de San Nicolás de Hidalgo (umsnh), Universidad Veracruzana, Centro de Investigación en Micro y Nanotecnología (uv-Microna), Instituto Tecnológico de Celaya (ITC), Instituto Tecnológico de Saltillo (ITS), Instituto Tecnológico de Hermosillo (Ітн), Instituto Tecnológico de Querétaro (ITQ), Instituto Politécnico de Pachuca (IPP), Instituto Tecnológico Superior de Irapuato (ITESI), Instituto Tecnológico de Estudios Superiores de Monterrey (ITESM), Universidad de Monterrey (UM), Universidad de las Américas Puebla (udla), Universidad Anáhuac México Sur (UAMs), Universidad Tecnológica de la Mixteca (UTM), Universidad Politécnica de Chiapas (UPCH), Universidad Autónoma de Zacatecas (UAZ) y el Instituto Tecnológico de Zacatepec (ITZ).

Algunas de estas instituciones ofrecen entrenamiento, en la forma de grados académicos de diferente nivel, relacionados con la nanotecnología y la nanociencia. No obstante, hay que recordar que la nanotecnología es una tecnología multidisciplinaria, por tal circunstancia, conocer exactamente el énfasis curricular de cada programa se convierte en una labor exhaustiva. Sin embargo, para este caso se presume que estas instituciones mantienen una orientación, o al menos especialización, temática en nanotecnología o nanociencia. En el cuadro 5 se presentan las instituciones y el número de grados académicos que ofrecen en relación con la nanotecnología.

Se observa que se repite el patrón de concentración de recursos e infraestructura. La UNAM es la institución que aglutina el mayor número de grados académicos directamente relacionados con la nanotecnología. De igual manera, el IPN y el Cinvestav son instituciones importantes en la formación de recursos humanos especializados en esta tecnología.

A pesar del número amplio de instituciones que supuestamente trabajan en nanotecnología, y como resultado de la falta de recursos financieros y de la presión por parte de la política de C\&T para contribuir al incremento de la competitividad, el grueso de las instituciones ha buscado formar acuerdos de cooperación y asociaciones. Existen varios acuerdos y perspectivas para formar alianzas con socios académicos y empresariales, tanto nacionales como extranjeros.

La cercanía geográfica con Estados Unidos y las coyunturas económicas de tal situación, como la firma del Tratado de Libre Comercio de 


\section{Cuadro 5}

Instituciones y número de grados académicos relacionados con la nanotecnología

\begin{tabular}{lccc}
\hline \multicolumn{1}{c}{ Institución } & Licenciatura & Maestría & Doctorado \\
\hline UNAM (incluye institutos y facultades) & - & 10 & 10 \\
IPN (incluye Cinvestav) & - & 6 & 5 \\
UASLP & 3 & 4 & 5 \\
Universidad de Sonora & - & 5 & 3 \\
Cimav & - & 2 & 2 \\
UAM (todos los campus) & - & 1 & 2 \\
Ipicyt & - & 1 & 2 \\
IMP & 1 & 1 & 1 \\
CIQA & - & 1 & 1 \\
UANL & - & 1 & 1 \\
UDLA & 2 & - & - \\
UG & - & 1 & 1 \\
\hline
\end{tabular}

Fuente: Elaboración propia y complementada con SE (2008).

América del Norte (TLCAN), han permitido que dicho país se conviertan en el principal aliado de México en I\&D de la nanotecnología. El agente promotor de las relaciones científicas entre las dos naciones es la Fundación México-Estados Unidos para la Ciencia (Fumec).

Una de las principales actividades de la Fumec en convenio con el Ministerio de Economía (2001), tiene como propósito facilitar la entrada en México del diseńo, desarrollo, empaque y comercialización de MEMs/ NEMS (Sistemas electromecánicos micro y nano). Para ello se creó la Red de Centros de Diseño mems donde participan 11 instituciones de educación superior mexicanas con el objetivo de diseñar MEMs/NEMs, desarrollar proyectos industriales y fortalecer los programas académicos especializados. También se formó el Centro de Articulación Productiva mems, en 2004, para facilitar la colaboración entre industria, academia y quienes toman las decisiones, para desarrollar nuevos productos y negocios. Una tercera actividad fue la creación de la Red de Laboratorios de Innovación, una cadena articulada de desarrollo de MEMs/NEMs donde los prototipos serían realizados en la UNAM y la UACJ, la manufactura en el laboratorio del INAOe en Puebla y el empaque en la UACJ en Chihuahua.

El Fumec también jugó un papel importante en la implementación del Laboratorio Binacional de Sustentabilidad (BNSL), una institución sin fines de lucro y no gubernamental que propone negocios tecnológicos a lo largo de la frontera. Comenzó sus actividades en 2005. Uno de sus principales asociados son los Sandia National Laboratories, laboratorios militares estadounidenses con sede en la ciudad de Albuquerque, Nuevo México. Estos sitios trabajan con la filosofía de grupo de concepto 
avanzado, que tiene el propósito de impulsar el desarrollo sustentable en la región fronteriza, reduciendo el estrés y aumentando las capacidades locales y la expansión empresarial. Las áreas estratégicas que definió son: empaque de mems que apoye el Paso del Norte Packaging Cluster en investigación y desarrollo de productos y su comercialización, energía y materiales avanzados para abaratar los costos en la zona fronteriza, y agua con desarrollos tecnológicos apropiados para la región. En todos los casos participan diversas instituciones científicas mexicanas, como la UACJ, el ITESM, el Cimav y empresas estadounidenses.

Además, el Fumec ha venido colaborando en varios workshops sobre nanotecnología, en acuerdos de intercambio académico y de estudiantes y otras actividades de enlace. En los últimos años se han unido representantes canadienses, lo que convierte algunos de los proyectos en trilaterales (Fumec, s/f).

Otras instituciones extranjeras e internacionales también tienen convenios de colaboración en nanotecnología con instituciones mexicanas. El Internacional Center for Nanotechnology and Advance Materials (ICNAM) es un consorcio resultado del convenio entre la Universidad de Texas-Austin y el Cimav en la ciudad de Chihuahua, realizado en 2003. El propósito es promover las investigaciones conjuntas binacionales, el intercambio de profesores e investigadores y alumnos. Por la parte mexicana el consorcio integra a la UASLP, el ITESM, la UNAM, la UANL, el Ipicyt, el CIQA, el Cinvestav y el propio Cimav (UTexas-A., s/f).

Por su parte, en 2005 el Cimav firmó un acuerdo con el UAlbany College of Nanoscale Science and Engineering de Albano NanoTech, para colaborar en investigación y educación en nanociencias. El acuerdo se centra en las áreas de optoelectrónica, nanofotónica, sensores químicos y biológicos, nanoestructuras moleculares y de carbono, nanopartículas y simulación computacional y modelación de nanoestructuras y nanosistemas (Azonano.com, 2005).

El mismo Cimav es sede, desde 2004, del Consorcio para Proyectos de Nanotecnología, que es parte de la iniciativa del Sistema Nacional de Redes de Grupos y Centros de Investigación del Conacyt. También participa en este consorcio el Ipicyt, el CIQA, el Cicese, el INAOE, además de varias empresas (Cementos de Chihuahua, Peñoles, Delphi, Lexmark, Mabe y Cydsa) (Cimav, 2004; Conacyt, 2006b).

El gobierno de Texas pretende colocar a este estado como líder mundial en el desarrollo de aplicaciones de nanotecnología. Para tal propósito busca impulsar varias asociaciones. Una de ellas es la Asociación Estratégica para la Investigación en Nanotecnología (Spring), una red entre UT-Austin, UT-Dallas y UT-Arlington, en la que también participa la Rice University de Atlanta y The Air Force Research Laboratory en Dayton, Ohio. Con 
este consorcio se cruzan otros, como Nano at the Border, en el cual participan algunas unidades de la Universidad de Texas (Arlington, Austin, Brownsville, Dallas y Pan American) y se creó para facilitar el intercambio académico y compartir instalaciones de investigación en nanotecnología, como el Center for Nano and Molecular Science and Technology de UTAustin. Nano at the Border tiene el propósito de integrar estudiantes y recursos humanos de la comunidad hispana (CNM, s/f).

Estos consorcios y partnerships facilitan acuerdos con universidades mexicanas directamente o mediante el Conacyt. Este es el caso entre el ICNAM y el Cimav en la ciudad de Chihuahua que ya mencionamos; o entre la UTexas-Dallas, que desde su NanoTech Institute ha establecido colaboraciones con la Universidad de Guanajuato, comenzando con el seminario Nanociencias para Aplicaciones Avanzadas, para establecer disciplinas y estancias de investigación de varios estudiantes mexicanos en la uT-Dallas (uTDallas, 2006).

Uno de los contactos más promisorios entre académicos y estudiantes de áreas científicas y de ingeniería de América Latina y Estados Unidos es el proyecto Pan American Advanced Study Institutes (PASI). Este proyecto se describe como

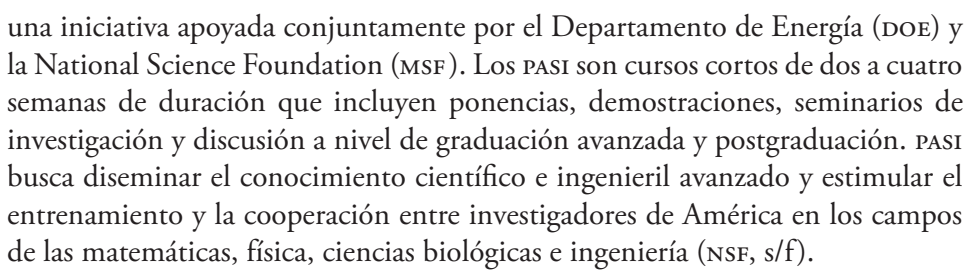

En el ańo 2001 se realizó en Costa Rica el primer Pasi dedicado a nanociencias (Physics and Technology at the Nanometer Scale) (Ulloa, 2002). Por lo menos otros ocho PASI sobre temas de nanotecnología se realizaron en diferentes países de América Latina. México participó con conferencistas y ponencias en varios, sino es que en todos ellos. Estos seminarios crean las condiciones para que los estudiantes y académicos puedan conocerse personalmente e intercambiar conocimientos y experiencias.

Existen varios acuerdos de colaboración con otros países y regiones. Por ejemplo, en 2004 se firmó el Acuerdo de Cooperación Científica y Tecnológica entre la Comunidad Europea y México, que entró en vigor en 2005. Este convenio posibilita la participación de centros de investigación mexicanos en las actividades del vi (2002-2006) Programa Marco de Desarrollo Tecnológico de la Unión Europea. La nanotecnología es un tema prioritario. Esto se refrendó con la creación de una oficina de enlace del Programa de Cooperación entre la Unión Europea y México en 
Ciencia y Tecnología (Uemexcyt), actualmente la oficina funciona con el plan de acción del Séptimo Programa Marco 2007-2013 (Uemexcyt, s/f).

También hay un convenio bilateral (Probicyt) de C\&T para fortalecer un sistema nacional de innovación, mejorar los recursos humanos y de infraestructura y promover la competitividad. México, junto con otros países latinoamericanos, participa en los programas @Lis (sociedad de la información), AL-Invest (inversiones), Alban (educación) y Urbal (desarrollo urbano) (Berlanga, 2004). Nanoforumeula es un proyecto de colaboración en I\&D en nanotecnología específicamente. En este consorcio participan la Universidad de Twente, Holanda; la Superintendencia de la Zona Franca de Manaus (Suframa), Brasil; el CEA-LETi-Minatec de Grenoble, Francia; el Fraunhofer IEs Institute de Desden, Alemania; la Universidad Autónoma de Madrid, España, y el Ipiciyt de México. Este proyecto es parte del interés de la Unión Europea por fomentar las relaciones de investigación a largo plazo entre instituciones europeas y de América Latina (Suframa, 2007).

El ciAm (Colaboración Interamericana en Materiales Avanzados) es un programa interinstitucional creado en 2002 para apoyar la colaboración en investigación de materiales y creación de redes. Participan las instituciones de C\&T de Argentina, Brasil, Canadá, Chile, Colombia, México, Jamaica, Trinidad y Tobago y Estados Unidos. Investigadores mexicanos han recibido varios financiamientos de esta organización para estudiar nanotecnología.

Se puede decir que varias universidades y centros de investigación están vinculados a la I\&D de la nanotecnología en México. No obstante, también prevalece una importante inequidad entre los centros y universidades más avanzadas y los que no tienen tanto protagonismo en el mundo nano de México. Otro aspecto a resaltar es el hecho de que a pesar de que no existe una iniciativa nacional en nanotecnología, la política de C\&T comprometida con la economía del conocimiento y con la búsqueda de competitividad ha logrado permear las estrategias y objetivos de los principales actores en la I\&D de la nanotecnología en el país. Existe, por consiguiente, el riesgo de que la búsqueda de competitividad termine exacerbando las diferencias entre los laboratorios mexicanos.

\section{Parques industriales y empresas en México vinculadas a la nanotecnología}

México ha seguido un proceso de clusterización del conocimiento nanotecnológico, con la intención de articularlo con las cadenas productivas de alto nivel, tanto nacionales como internacionales. Para tal propósito se han implementado planes para crear parques industriales de alta tecno- 
logía. Se trata de espacios físicos con infraestructura y condiciones para la instalación de empresas nacionales y transnacionales, apoyadas por centros de investigación de alta tecnología. Pero el éxito de estos parques en México requiere revertir la tendencia de salida del país de empresas manufactureras, que ya tiene, por lo menos, una década en México.

En el ámbito mundial, diversos avances técnicos en los últimos 20 años -como las telecomunicaciones, los sistemas de almacenamiento y transporte de mercadería más eficientes, y también regulaciones menos estrictas en cuestiones de medio ambiente o laborales- han permitido abaratar precios y que empresas antes instaladas en Estados Unidos, por ejemplo, migraran a países del sudeste asiático. Esta tendencia incluye personal calificado y actividades de diseńo de prototipos e incluso la producción propiamente dicha. Mientras que países como China, Tailandia y Singapur han aumentado la contribución de las manufacturas como porcentaje del producto interno bruto desde 1990, tanto Estados Unidos como México han disminuido esta participación (Hung y León, 2005). Esta tendencia pone en riesgo la instalación de parques industriales de C\&T; más aún si se consideran que reciben los técnicos y científicos por migrar (fuga de cerebros) y la falta de formación científica en C\&T de vanguardia en la educación primaria, secundaria y universitaria, que hace que los centros de posgrado en nanotecnología en México puedan no contar con la oferta necesaria en cantidad y calidad. ${ }^{1}$ No obstante, hay claros ejemplos de que la política de parques industriales de alta tecnología es una estrategia consistente de acuerdo con las convicciones del gobierno de México.

El Silicon Border Development Science Park (SBDSP) se promociona como el primer parque de alta tecnología especializado en nanocomponentes de América Latina. Se ubica en Mexicali, Baja California, y abarca 400 hectáreas. El propósito es crear un parque científico que abastezca toda la cadena de la industria de semiconductores y otras de alta tecnología. El sBDSP cuenta con el apoyo del gobierno federal y del estado de Baja California, por parte de México, y del gobierno de California por parte de Estados Unidos (sBDSp, s/f). El proyecto para construir el parque comenzó en 2006 y se espera que se concluya en 10 años (TMC.net, 2005).

El gobierno del estado de Puebla tiene la intención de crear un parque de alta tecnología en Huejotzingo. Se espera que pueda ofrecer productos

${ }^{1}$ En un encuentro organizado por el Banco Mundial en febrero del 2007, el presidente del Banco urgió a los países en desarrollo a apoyar la ciencia, pero dijo que había que ser cuidadosos ya que "El peligro actual yace en promover políticas que ven a la ciencia y tecnología como conductores de progreso social y económico, antes que como componentes de programas de innovación en los cuales otros factores -desde política regulatoria a la educación y entrenamiento- son igual de importantes" (SciDevNet, 2007). 
para la industria médica y automovilística (García, 2007). Se pretende aprovechar la coyuntura de la presencia del INAOE y la posibilidad de vincular al LNN al circuito de producción de MEMS/NEMS. Hasta ahora está pendiente el apoyo esperado por parte de la Secretaría de Economía. De igual manera, se desarrolla el Parque Tecnológico CIт (Centro de Innovación y Transferencia) de Puebla, con el apoyo directo del ITESM en el estado. La idea es fomentar la innovación que permita mejorar la competitividad de las cadenas productivas de la región (CIT-ITESM, s/f).

A partir de 2005 se construye en el estado de Nuevo León, el Parque de Investigación e Innovación Tecnológica (РIIT). Este parque es parte del proyecto Ciudad del Conocimiento, donde se pretende atraer los principales centros de educativos y de investigación de México a la ciudad de Monterrey. La UANL participa de este proyecto con el Centro de Innovación, Investigación y Desarrollo en Ingeniería y Tecnología, donde se ubica el Laboratorio de Nanotecnología y Nanociencias. Otras instituciones de investigación ya están presentes en el parque. El Conacyt tiene una subsede del Centro de Ingeniería y Desarrollo Industrial y el CIMAV con un subcentro del Nanotech; el ITESM tiene el Centro de Investigación y Diseño Estratégico de Producto (CIDEP); la UAEM tiene el Centro de Empaquetotecnia Avanzada; el IPN llevará el Cinvestav; el Instituto del Agua de Nuevo León también estará presente; varias empresas de software formarán un cluster; se creará una incubadora de empresas y también se instalarán otras empresas como Sigma, Pepsico y Motorola. Actualmente, el PIIT se prepara para ser sede del Congreso Latinoamericano de Parques Científicos y Tecnológicos, que tiene como objetivo intercambiar las experiencias respecto a las incubadoras de empresas de alta tecnología (PIIT, 2009).

The Paso del Norte mems/Nems Packaging Cluster tiene como objetivo crear un corredor tecnológico entre Albuquerque, Nuevo México, y la ciudad de Chihuahua. Entremedio se ubican Ciudad Juárez y El Paso, con la UACJ y la UT-El Paso jugando roles clave en términos científicos en el cluster. Éste incluye centros de investigación, empresas y los laboratorios militares Sandia y cuenta con el apoyo de la Fumec.

También existen otros acuerdos que hacen viable una vinculación entre el sector científico-productivo en México y la industria militar. Hay poca información al respecto ya que la mayor parte de estos documentos son clasificados. Empero, de los acuerdos militares que se han hecho públicos destaca la firma, en 2005, del tratado Security and Prosperity Partnership of North America (sppna) entre México, Estados Unidos y Canadá, y en el marco del Tratado de Libre Comercio de América del Norte. El tratado incluye la colaboración científica en I\&D en diversas áreas, incluida la nanotecnología, en un marco directamente influenciado por los sectores 
militares (sPPNA, 2005). De esta forma se busca insertar parte de la I\&D de la nanotecnología mexicana al mercado de la industria militar, sobre todo en relación con nuevos materiales y compuestos suplementarios.

Las empresas que trabajan con nanotecnología en México quedan como una incógnita, ya que no existe base de datos, observatorio o ley que regule empresas que trabajen o desarrollen aplicaciones a partir de la nanotecnología. En el cuadro 6 se exponen algunos ejemplos.

En este cuado se mencionan 23 empresas, recopiladas hasta abril de 2009, que utilizan insumos nanotecnológicos o que los obtienen mediante síntesis en sus laboratorios. Los productos finales en su uso también aplican nanotecnología o utilizan las ventajas que los insumos nanotecnológicos les proporcionan.

También es interesante analizar en este cuadro la tendencia desigual en la distribución geográfica del origen de cada empresa. Encontramos que siete empresas surgen en la ciudad de México y que otras siete están en el estado de Nuevo León, las entidades federativas uno y dos, respectivamente, en la clasificación nacional de estados competitivitos (Imco, 2008). Hay una en Coahuila, otra en el estado de Jalisco y una paraestatal nacional, Pemex. Las demás son parte de conglomerados extranjeros, principalmente de Estados Unidos, aunque se encuentra una empresa de Japón y otra de Alemania. También resalta el hecho de que la gran mayoría utiliza como insumo nanopartículas, sea producido por estas empresas o adquirido por un proveedor determinado.

\section{Conclusiones}

Hemos observado la manera en que la nanotecnología se inserta en el proceso de desarrollo capitalista de México. En este contexto, encontramos que, ante la ausencia de una iniciativa nacional, la nanotecnología pareciera tener varias direcciones. Pero a pesar de que no existe un orden claro, es evidente que la I\&D en México la están moldeando las relaciones de competencia en el mercado.

La nanotecnología promete ser la próxima revolución industrial, de manera que aquellos países que no logren incorporarse podrían quedar fuera de la nueva redistribución industrial resultante de la competencia. Además, la economía mundial, basada cada vez más en el conocimiento y en la innovación tecnológica, coloca en desventaja a aquellos países que no invierten en educación y conocimiento. En este contexto la nanotecnología aparece como una obligación, en lugar de una opción, y México ha comprado esa receta de desarrollo.

Pero al mismo tiempo México se encuentra muy atrás en relación con otros países, industrializados y emergentes, con respecto a los recursos 


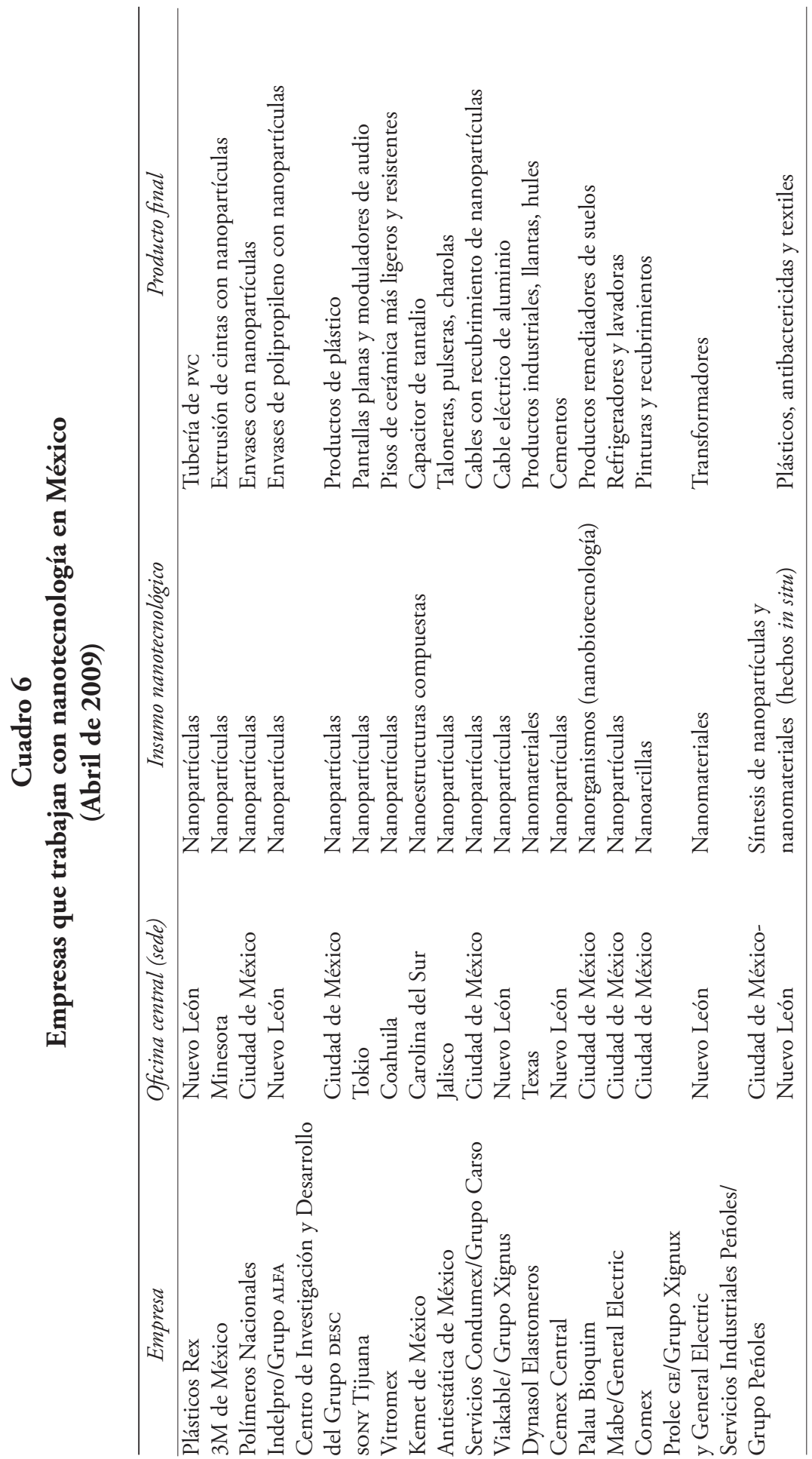




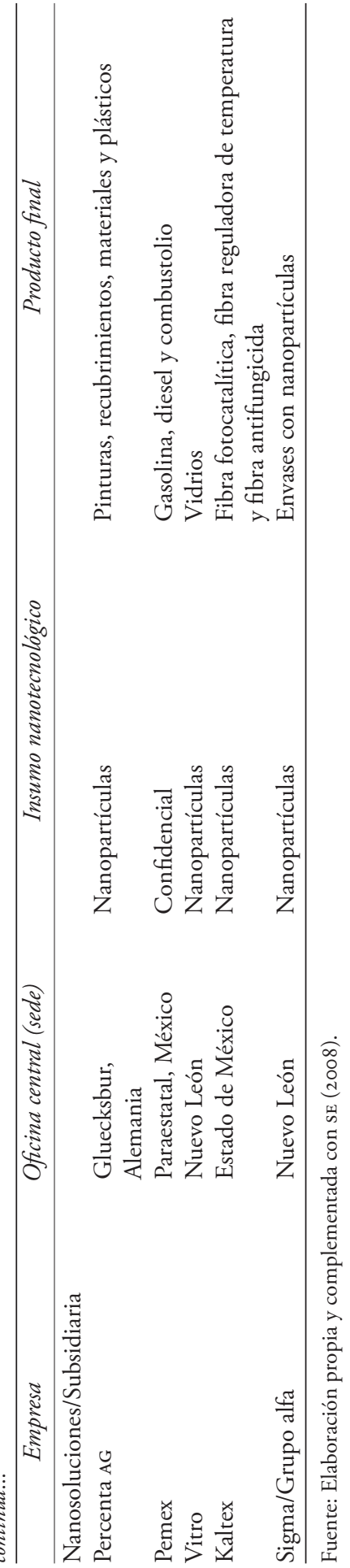


destinados a la C\&T en general y a la nanotecnología en particular. Esto también ha repercutido negativamente en el patentado de los desarrollos nanotecnológicos. Pero incluso, en el caso poco probable que se puedan igualar los presupuestos de los grandes jugadores de la nanotecnología, quedaría por ver si el hecho de que las empresas sean más competitivas se puede traducir en la reducción de la pobreza y la inequidad; ya que una mejoría en la competitividad y en la macroeconomía se traduce en la reducción de la inequidad.

De lo anterior surge la preocupación que tiene que ver con la cantidad y calidad de recursos humanos que se requieren para desarrollar la nanotecnología. Resulta poco probable que, cuando la mitad de la población mexicana vive en pobreza y cuando son muy pocos los que terminan grados superiores universitarios, se pueda proveer del personal que los parques industriales y laboratorios de alto nivel requerirán. También es necesario pensar que ante la ausencia de una dirección nacional en nanotecnología se corre el riesgo de que los esfuerzos duplicados y desordenados, así como la competencia entre los actores domésticos en nanotecnología terminen por ampliar la ya de por sí exacerbada inequidad. La situación de pobreza entre los mexicanos se refleja de igual manera en la inequidad de recursos e infraestructura entre los centros, universidades, laboratorios y empresas que trabajan con nanotecnología.

Finalmente, la falta de atención a los efectos políticos, sociales, económicos y ambientales de la nanotecnología también es preocupante. La ausencia de regulación es un espacio de oportunidad para actores de otros países que no pueden investigar o promover ciertos proyectos nanotecnológicos en sus países; pero sus consecuencias a la salud y ambientales son inciertas.

\section{Bibliografía}

Azonano.com (2005), U-Albany NanoTech College Signs Agreement With Mexico's Leading Materials Laboratory Fostering Research and Student Exchange, Recent News, www.azonano.com/news. asp?newsID=847, 13 de junio de 2009.

Bawa, Raj, Raji Bawa, Stephen Maebius, Ted Flynn y Chiming Wei (2005), "Protecting new ideas and inventions in nanomedicine with patents", Nanomedicine: Nanotechnology, Biology, and Medicine, I, Elsevier, Ámsterdam, pp. 150-158.

Berlanga Subyaga, Claudia (2004), Cooperación ciencia y tecnología Unión Europea-México, presentación en Power Point, www.cudi. 
edu.mx/primavera_2004/presentaciones/CLaudia_Berlanga.pdf, 17 de marzo de 2007.

вм (Banco Mundial) (2007), Building Knowledge Economies, Advance Strategies for Development, Banco Mundial, Washington.

Cenam (Centro Nacional de Metrología) (s/fa), Servicios y objetivos, www.cenam.mx/servicios/, 18 de junio de 2009.

Cenam (Centro Nacional de Metrología) (s/fb), Objetives Tri-national Workshop on Standards for Nanotechnologies 2009, www.cenam. $\mathrm{mx} /$ servicios/, 18 de junio de 2009 .

Científica (2007), Half Way to the Trillion Dollar Market?, cientifica.eu/ whitepapers/halfwaytotrilliondollar, 16 de junio de 2009.

Científica (2009), The Nanotechnology Opportunity Report, 2009, cientifica.eu/index.php?option=com_virtuemart\&page=shop. product_details\&flypage $=$ shop.flypage $\&$ product_id $=3,3 \mathrm{de}$ marzo de 2009.

Cimav (Centro de Investigación en Materiales Avanzados) (2004), Anuario Cimav 2004, Cimav-Conacyt www.conacyt.mx/Centros/Anuarios/2004/ANUARIO-2004-CIMAV.pdf, 20 de junio de 2009.

Cinvestav (Centro de Investigaciones y de Estudios Avanzados) (s/f), Maestría y doctorado en nanociencias y nanotecnología, cori. cinvestav.mx/Nano/, 16 de junio de 2009.

CiT-ITESm (Centro de Innovación y Transferencia-Instituto Tecnológico y de Estudios Superiores de Monterrey de Puebla) (s/f), Centro de Innovación, web.pue.itesm.mx/pt-cit/cit.html, 22 de junio de 2009.

CNM (Center for Nano and Molecular Science and Technology) (s/f), About CNM, www.cnm.utexas.edu/partnerships_nanoatborders. htm, 21 de junio de 2009.

Conacyt (Consejo Nacional de Ciencia y Tecnología) (2006a), Resultados de la Convocatoria de Apoyos Complementarios para el Establecimiento de Laboratorios Nacionales de Infraestructura Científica o Desarrollo Tecnológico, www.conacyt.mx/FONDOS/Institu- 
cional/LaboratoriosNacionales/Resultados_Convocatoria_Laboratorios_Nacionales_2006.pdf, 3 de junio de 2009.

Conacyt (Consejo Nacional de Ciencia y Tecnología) (2006b), Informe General del Estado de la Ciencia y Tecnología, México, www. siicyt.gob.mx/siicyt/docs/contenido/IGECyT_2006.pdf, 20 de junio de 2009.

Conacyt (Consejo Nacional de Ciencia y Tecnología) (2007), Indicadores de Actividades Científicas y Tecnológicas, www.siicyt.gob.mx/ siicyt/publicaciones/MostrarPublicacionesAction.do?anio=2007, 20 de junio de 2009.

Coneval (Consejo Nacional de Evaluación de la Política Social) (2008), Informe de evaluación de la política de desarrollo social en México 2008, www.coneval.gob.mx/coneval2/htmls/sala_prensa/ HomeSalaPrensa.jsp?id=informe_evaluacion_2008, 14 de junio de 2009.

Delgado Wise, Raúl y Noela Invernizzi (2002), "México y Corea del Sur: claroscuros del crecimiento exportador en el contexto del globalismo neoliberal", Aportes, Revista Mexicana de Estudios sobre la Cuenca del Pacifico, II $(2,4)$, Universidad de Colima, Colima, pp. 63-86.

DOF (Diario Oficial de la Federación), Decreto por el que se reforman, adicionan y derogan diversas disposiciones de la Ley de Ciencia y Tecnología, dof.gob.mx/nota_detalle.php?codigo $=5094366 \&$ fecha, 12 de junio de 2009.

Foladori, Guillermo (2006), "Nanotechnology in Latin America at the Crossroads", Nanotechnology Law \& Business Journal, 3 (2), NLB, Pasadena, pp. 205-216.

Fumec (Fundación México-Estados Unidos para la Ciencia) (s/f), Biennial Activities Report 2004-2005, www.fumec.org.mx/ingles/annual/ informe_completo_eng.pdf, 12 de febrero de 2009.

García León, Mauricio (2007), "Una Realidad, el Clúster Tecnológico en Puebla", Intolerancia, Puebla, http://209.85.7.40/ wwwinto/ cgibin $/$ cgis $/$ Seccion.pl?id_seccion=12\&anio $=2006 \& \mathrm{mes}=9 \& \mathrm{dia}$ =7\&pagina=1, 22 de abril de 2008 . 
Hung, Wayne y Jorge León (2005), "Micromanufacturing to Bridge Macro and Nano Worlds. Conference Presentation: sme/cirp International", Conference on Manufacturing Engineering Education, 22-25 de junio de 2005, Cal Poly State University, California, mnmlab.tamu.edu/publication/MfEE_Hung_Leon_21Mayo05. pdf, 12 de mayo de 2007.

Insead (Institute Européen d'Administration des Affaires) (2009), Global Innovation Index 2008-2009, www.enter.es/enter/cms/es/documento/5786/1, 4 de mayo de 2009.

Imco (Instituto Mexicano para la Competitividad) (2008), Aspiraciones y realidad, las agendas del futuro: índice de competitividad estatal, www.imco.org.mx/imco/recursos/webestados/home.html, 23 de junio de 2009.

IMP (Instituto Mexicano del Petróleo) (s/f), Áreas temáticas de investigación en posgrado, www.imp.mx/investigacion/posgrado/?imp=areas, 15 de junio de 2009.

INAOE (Instituto Nacional de Astrofísica, Óptica y Electrónica) (s/f), Laboratorio Nacional de Nanoelectrónica, www-elec.inaoep.mx/ $\operatorname{lnn} /$ index.php, 16 de junio de 2009.

Knowledge for Development Program (K4D) (s/f), Knowledge for Development, web.worldbank.org/WBSITE/EXTERNAL/ WBI/WBIPROGRAMS/KFDLP/0,,contentMDK:20269026 menuPK:461205 -pagePK:64156158 -piPK:64152884 -theSite PK:461198,00.html\#Knowledge, 4 de mayo de 2009.

KEI (Knowledge Economy Index) (s/f), www.worldbank.org/kam, 16 de junio de 2009.

Laboratorio Nacional de Investigaciones en Nanociencias y Nanotecnología (Linan), Objetivos y plan de desarrollo, http://materials. ipicyt.edu.mx/LINAN/, 10 de junio de 2009.

Lopéz, Jose Antonio y Nayelli Roldán (2009), "El príncipe de Asturias, nuevo galardón de la unam", Milenio Diario, http://impreso. milenio.com/node/8589928, 12 de junio de 2009. 
Miller, Georgia y Rye Senjen (2008), Out of the laboratory and into the food chain: Nanotechnology in food and agricultura, Friends of Earth-Australia, www.foe.org/pdf/nano_food.pdf, 10 de junio de 2009.

Nanotech (Laboratorio Nacional de Nanotecnología) (s/f), Bienvenida, nanotech.cimav.edu.mx/, 9 de junio de 2009.

NASA (Administración Nacional de Aeronáutica y del Espacio) (s/f), Why Nanotechnology at nasa?, www.ipt.arc.nasa.gov/nanotechnology. html, 20 de junio de 2009.

NNI (National Nanotechnology Initiative) (2009), Research and Development Leading to a Revolution in Technology and Industry, Suplemment to the President's fyi 2010 Budget, www.nano.gov/, 20 de junio de 2009.

NSF (National Science Foundation) (s/f), Pan-American Advanced Studies Institutes Program (PASI), www.nsf.gov/funding/pgm_summ. jsp?pims_id=5327, 20 de junio de 2009.

OCDE (Organización para la Cooperación y el Desarrollo Económico) (2002), "Propuesta de norma práctica para encuestas de investigación y desarrollo experimental", OCDE, París, www.oecdbookshop. org/oecd $/$ display.asp?lang=EN\&sf $1=$ identifiers\&st $1=92200208$ $1 \mathrm{p} 1,4$ de marzo de 2009.

oCDE (Organización para la Cooperación y el Desarrollo Económico) (2008), oECD in Figures, dx.doi.org/10.1787/467638774024, 8 de mayo de 2009.

ocDe (Organización para la Cooperación y el Desarrollo Económico) (2009), Reviews of Regional Innovation: 15 Mexican Status, OCDE, París.

OEI (Organización de Estados Iberoamericanos) (2007), La nanotecnología en Iberoamérica. Situación actual y tendencias, www.oei. es/observatoriocts/index.php?option=com_content\&view=articl e\&id=12\&Itemid=3, 7 de mayo de 2009.

ONCN (Oficina Nacional de Coordinación de la Nanotecnología) (2009), Nanotechnology, Big Things From a Tiny World, www.nano. 
gov/html/res/200711NanotechnologyBigThingsfromaTinyWor ld.html, 18 de septiembre de 2009.

OMPI, Estadísticas de Patentes, 2008, www.wipo.int/ipstats/es/statistics/ patents/, 14 de marzo de 2009.

PIIT (Parque de Investigación e Innovación Tecnológica) (2009), Conferencia Latinoamericana de Parques Científicos y Tecnológicos, www.iasp.ws/publico/index.jsp?enl=5, 23 de junio de 2009 .

pecyt (Programa Especial de Ciencia y Tecnología 2001-2006) (2001), www.siicyt.gob.mx/siicyt/docs/Programa_Nacional_ de_C_y_T_1970-2006/documentos/PECYT.pdf, 3 de junio de 2009.

PeCYT (Programa Especial de Ciencia y Tecnología 2008-2012) (2008), www.conacyt.mx/Acerca/Normatividad/Programa-Especial-deCiencia-y-Tecnologia_2008-2012.pdf, 13 de mayo de 2009.

Punta (Proyecto Universitario de Nanotecnología) (2004), Proyecto Universitario de Nanotecnología, inter.fciencias.unam.mx/punta/ index.php?option $=$ com_content $\&$ task $=$ category \&sectionid $=5 \&$ $\mathrm{id}=22 \&$ Itemid $=39,11$ de junio de 2009.

Quezada Barrón, Manuel (2009), "Crean centro trinacional de nanotecnología”, Biz News, Chihuahua, México, biznews.com.mx/cms/ index.php?option $=$ com_content $\&$ view $=$ article $\& i d=68$ : nanotec nologia-chihuahua\&catid=45:tecnologia, 10 de junio de 2009 .

Regina (Red de Grupos de Investigación en Nanociencias) (s/f), Página de bienvenida, www.nano.unam.mx/index.php?option=com frontpage\&Itemid=76, 13 de junio de 2009 .

Robles-Belmont, Eduardo, David Vinck y Rebeca de Gortari Rabiola (2008), "Desarrollo de las nanociencias en México: una visión a partir de las publicaciones científicas", hal.archives-ouvertes. fr/docs/00/36/25/94/PDF/NanoMex08_Robles_1_.pdf, 4 de junio de 2009.

RS\&RAE (Royal Society and Royal Academy of Engineering) (2004), Nanoscience and Nanotechnologies: Opportunities and Uncer- 
tainties, Policy document 20/04, Londres: rs\&rae, www.nanotec. org.uk/finalReport.htm, 20 de junio de 2009.

Ruíz, Rosaura (2009), "El financiamiento público para la C\&T en el presupuesto de egresos de la federación para 2009", www.comunicacion.amc.edu.mx/comunicacion/docs/amc-rrg-051208pres2009.pdf, 9 de mayo de 2009.

SE (Secretaría de Economía) (2008), Diagnóstico y prospectiva de la nanotecnología en México, www.economia.gob.mx/pics/pages/944_base/Nanotecnologia.pdf, 1 de abril de 2009.

SCI (Science Citation Index) adaptado desde E. Robles-Belmont, David Vinck y Rebeca de Gortari Rabiola, Desarrollo de las nanociencias en México: una visión a partir de las publicaciones científicas, 2008, hal.archives-ouvertes.fr/docs/00/36/25/94/PDF/ NanoMex08_Robles_1_.pdf, 4 de junio de 2009.

SciDevNet (Science and Development Network) (2007), "Investing in science: a cautionary tale", www.scidev.net/Editorials/index.cf $\mathrm{m}$ ?fuseaction=readEditorials\&itemid $=211$ \&language $=1,22 \mathrm{de}$ marzo de 2007.

SBDSP (Silicon Border Development Science Park), Government Support, www.siliconborder.com/Government_support.html, 23 de junio de 2009.

sppna (Security and Prosperity Partnership of North America) (2005), Report to the Leaders. Prosperity Annex, www.spp.gov/report to_leaders/prosperity_annex.pdf?dName=report_to_leaders.pdf, 22 de junio de 2009.

Suframa (Superintendencia de la Zona Franca de Manaus) (2007), "Nanoforumeula: colaboraciones de investigación y desarrollo en nanotecnología entre la Unión Europea y América Latina", Minapim News, 9, www.suframa.gov.br/minapim/news/visArtigo. cfm?Ident=374\&Lang=ES, 16 de febrero de 2007.

TMc.Net (2005), "Silicon Border Breaks Ground in Mexicali: Science Park Becomes a Reality", www.tmcnet.com/usubmit/2005/ jul/1162162.htm, 10 de diciembre de 2009. 
Uemexcyt (Unión Europea y México en Ciencia y Tecnología) (s/f), Acuerdo de Cooperación entre la Unión Europea y México en Ciencia y Tecnología, www.conacyt.gob.mx/uemexcyt/portada. html, 22 de junio de 2009.

Ulloa, Sergio Eduardo (2002), "Nanoscience in Latin America", Journal of Nanoparticle Research, 4 (3), Springer, Dordrecht, pp. 175-177.

Undp (United Nations Development Program) (2008), Human Development Report 2008, undP, Nueva York.

UTDallas Community (2006), Nano at the Border, www.nanotech.utdallas.edu/community/natb/index.html, 21 de junio de 2009.

uTexas-A (s/f), International Center for Nanotechnology and Advance Materials, www.engr.utexas.edu/icnam/, 20 de junio de 2009.

wIPo (World Intelectual Property Organization) (2008), World Patent Report, A Statistical Review, www.wipo.int/ipstats/es/statistics/ patents/, 7 de junio de 2009 .

Recibido: 9 de julio de 2007. Reenviado: 3 de julio de 2009. Aceptado: 9 de septiembre de 2009.

Edgar Záyago-Lau. Es doctor en estudios del desarrollo por la Universidad Autónoma de Zacatecas (UAZ); es maestro en estudios del desarrollo internacional por la Universidad Saint Mary's en Halifax, Nueva Escocia, Canadá. Actualmente es investigador de la Red Latinoamericana de Nanotecnología y Sociedad (Relans). Sus líneas de investigación incluyen nanotecnología, desarrollo y sociedad y economía política del desarrollo. Entre sus publicaciones destacan: "La nanotecnología y el desarrollo económico", Milenio Diario, México, 13 de marzo (2007); "Nanotechnologies for Development in Latin America", en Guillermo Foladori y Noela Invernizzi (eds.), Nanotechnologies in Latin America, Rosa-Luxemburgo-Stiftung, Berlín (2008); "Nanotecnología y los enclaves del conocimiento en Latinoamérica", Estudios Sociales, XVII (34), CIAD, Hermosillo, pp. 327-348 (2009).

Guillermo Foladori. Es doctor en economía por la Universidad Nacional Autónoma de México; es especialista en medio ambiente y desarrollo, y salud y desarrollo. Ha trabajado como profesor en la UNAM, la Universidad 
Autónoma de Chapingo y la Escuela Nacional de Antropología e Historia (ENAH) en México. Entre sus publicaciones destacan: Controversias sobre sustentabilidad, Miguel Ángel Porrúa, México (2001); Limites do desenvolvimento sustentable, Unicamp, São Paulo (2001); Proletarios y campesinos, Universidad Veracruzana, Jalapa (1986); “¿Sustentabilidad? Desacuerdos sobre el desarrollo sustentable”, Miguel Ángel Porrúa, México (2001). Es miembro del Sistema Nacional de Investigadores (nivel III), Red Latinoamericana de Nanotecnología y Sociedad. 